\title{
RECENT ELECTROWEAK RESULTS FROM CDF
}

\author{
ALEXEI N. SAFONOV \\ (FOR CDF COLLABORATION) \\ Department of Physics, \\ Texas A\&M University, \\ College Station, TX 7r843, USA \\ E-mail: safonov@tamu.edu
}

We present recent CDF results in the area of electroweak physics based on 350-1000 $\mathrm{pb}^{-1}$ of Run II data.

\section{Intoduction}

Precision experimental studies of the electroweak processes are of utmost importance for understanding the mechanism of spontaneous symmetry breaking in the Standard Model (SM) and verifying the SM gauge structure predicted by the $S U(2) \times S U(2) \times U(1)$ symmetry group. Furthermore, these measurements provide an insight and indirect constraints on the properties of physics beyond our current direct reach. A perfect example is constraints arising from the $W$ mass measurement on the Higgs sector. There is a potential for discovery of new physics should a significant deviation in the measured quantities from the SM predictions be detected. Finally, precision measurements combined with other experimental data constrain parton distribution functions (PDFs), which is important in view of upcoming LHC experiments. CDF has a strong program of electroweak measurements, some of the highlights and their motivations are:

- Measurement of the $W$ mass: strong implications on the Higgs mass;

- Studies of the diboson production: a direct measurement of the trilinear couplings and sensitivity to new physics;

- Measurements of $W$ and $Z$ production cross-sections: a test of the higher order calcuations in the context of the SM;

- Studies of the $W$ and $Z$ production asymmetries: PDF constaints. 
In this paper, we describe recent $\mathrm{CDF}$ electroweak measurements and discuss their importance in the context of precision tests of the SM. The reader should be aware that $W$ mass results are reported as a separate contribution.

\section{Studies of Di-Boson Production}

Deviations of the trilinear couplings from the values predicted by the SM breaks the fine tuning in the SM that provides cancellation of the fast growing terms from contributing Feynmann diagrams, which may eventually violate unitarity. To prevent that, a new physics at the scale of a few $\mathrm{TeV}$ has to be introduced ${ }^{1}$. Therefore, deviation of the measured parameters of the trilenear couplings from their SM values would indicate presence of new physics. If that happens, current sensitivities are such that this new physics is likely to be accessible by upcoming LHC experiments.

\subsection{First Observation of the $W Z$ Production}

$\mathrm{CDF}$ has recently completed an analysis that yielded the first observation of $W Z$ production. This analysis uses $1 \mathrm{fb}^{-1}$ of data and targets events where both vector bosons decay via leptonic modes. Event selection requires three identified leptons (electrons or muons), the stiffest one satisfying $p_{T}>20$ $\mathrm{GeV} / c$ and remaining two have to have $p_{T}>10 \mathrm{GeV} / c$. To increase acceptance, several loose lepton categories are defined to compensate for partially instrumented regions of the detector. Next, selected events are required to further pass $\mathbb{E}_{T}>25 \mathrm{GeV}$, which virtually eliminates most of the backgrounds except $t \bar{t}$. At least one pair of same flavor and opposite sign leptons is required to have invariant mass in the range $[76,106] \mathrm{GeV} / c^{2}$ consistent with the $Z$ mass. To suppress $Z Z$ backgrounds, an additional requirement is applied that there is no additional track in the event with $p_{T}>8 \mathrm{GeV} / c$ that together with the third lepton would form an invariant mass consistent with the $Z$ boson mass. There are 16 events remaining after these selections with the expected background of $2.7 \pm 0.4$ events. The excess is consistent with the hypothesis of being $W Z$ events as demonstrated in Fig. 1a showing invariant mass of the two lepton candidates clearly indicating $Z$ bosons and the $\mathbb{F}_{T}$ disctribution (Fig. 1b) in the final events with excess consistent with the $W$ hypothesis. Significance of the excess is nearly 6

sigma. Measured cross-section is consistent with the SM expectation ${ }^{2}$ and is the first experimental observation of the $W Z$ production. 

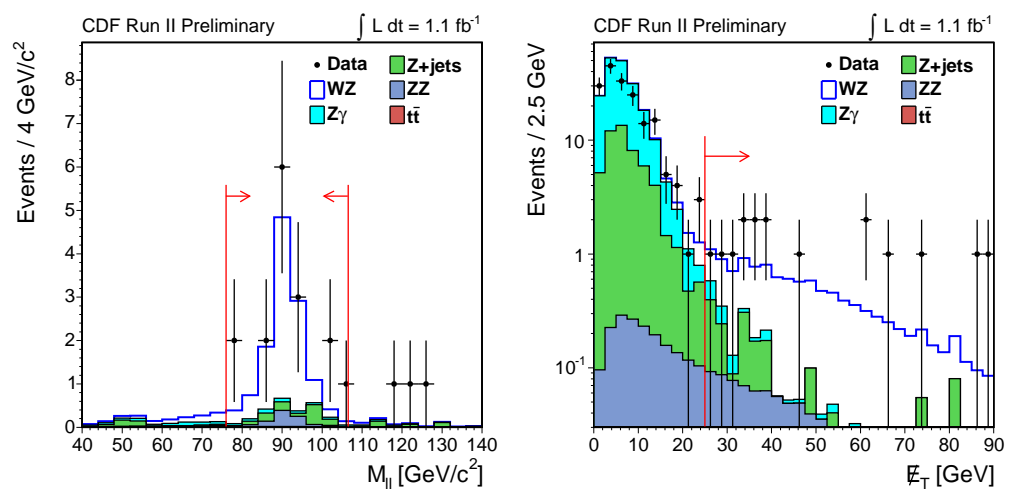

Figure 1. a): Invariant mass of the two lepton candidates; b) $\mathbb{E}_{T}$ distribution in $W Z$ candidate events.

\section{2. $Z_{\gamma}$ Production}

This measurement is based on about $1 \mathrm{fb}^{-1}$ of data and is performed for the channel where $Z$ decays to electrons. Event selection requires two well reconstructed electron candidates with $p_{T}>20 \mathrm{GeV} / c$ in central or forward region of the calorimeter and a central photon candidate with $p_{T}>7$ $\mathrm{GeV} / c$. To suppress backgrounds, cuts are applied on the invariant mass of two electrons $m(e e)>40 \mathrm{GeV} / c^{2}$ and of the eer system $m(e, e, \gamma)>40$ $\mathrm{GeV} / c$. Remaining events, see Fig. 2a) are dominated by the $Z_{\gamma}$ events, while the leading background is due to $Z+$ jet production where jet is misidentified as a photon. Event counting leads to the cross-section measurement of $\sigma(Z \gamma)=4.9 \pm 0.3$ (stat) \pm 0.3 (sys) \pm 0.3 (lum) pb consistent with the $\mathrm{SM}$ expectation ${ }^{3}$ of $4.7 \pm 0.4 \mathrm{pb}$.

\section{3. $W \gamma$ Production}

This measurement is using $W$ bosons decaying to $\mu \nu$. Event selection requires a muon with $p_{T}>20 \mathrm{GeV} / c^{2}$ and missing transverse energy $\mathbb{F}_{\Gamma}>25 \mathrm{GeV}$. Photon selections are similar to the $W \gamma$ analysis. To further improve purity of the data, the transverse mass of the muon and $\mathbb{F}_{T}$ is required to satisfy $30<m_{T}\left(\mu, \mathbb{F}_{T}\right)<120 \mathrm{GeV} / c^{2}$ consistent with the $W$-boson hypothesis. 855 events pass all selections, of which approximately 300 events are expected to be due to backgrounds dominated by $W+$ jet and $Z \gamma$ events. This yields a cross-section measurement of $\sigma(W \gamma)=19.1 \pm 1.0$ (stat) \pm 2.4 (sys) \pm 1.1 (lumi) pb consistent with the $\mathrm{SM}$ expectation ${ }^{4}$ of $19.3 \pm 1.4 \mathrm{pb}$. Figure $2 \mathrm{~b}$ ) shows transverse mass of the 

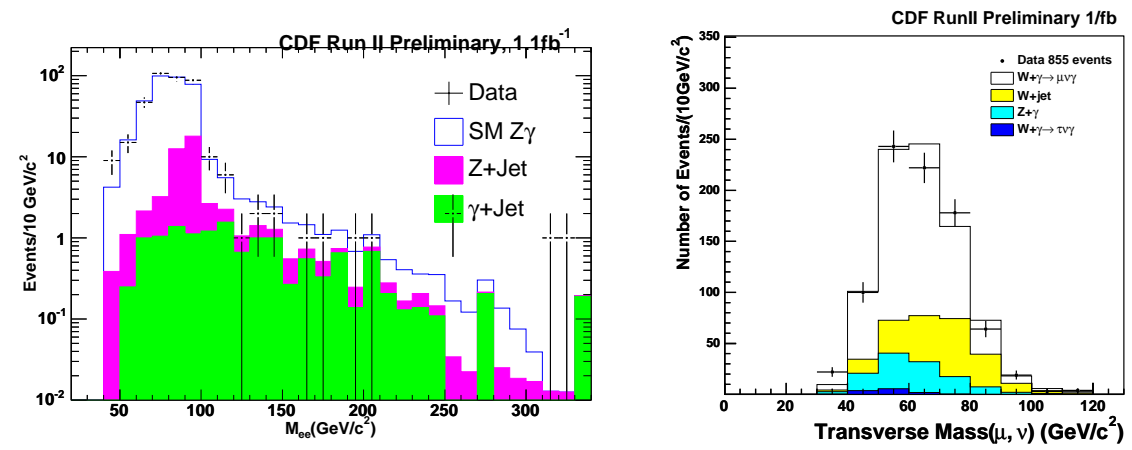

Figure 2. a): Invariant mass of the two lepton candidates in $Z \gamma$ candidate events; b) transverse mass of the $W \gamma$ candidate events.

candidate events.

\section{3. $W$ and $Z$ Production: $Z \rightarrow \tau \tau$}

CDF has completed measurements of the inclusive $W$ and $Z$ production cross-sections in electron and muon channels using $72 \mathrm{pb}^{-1}$ of data. Those results were found to be in good agreement with the NNLO expectations ${ }^{5}$. Recently, we have added the $Z \rightarrow \tau \tau$ channel, which is the first electroweak measurement using taus in Run II.

This analysis relies on the mode where one of the two taus decays into an electron channel while the other one decays hadronically. Analysis requires at least one good electron candidate with $p_{T}>10 \mathrm{GeV} / c$ and a tau candidate with $p_{T}>15 \mathrm{GeV} / c$. Two candidates have to have opposite sign charges. We then apply cuts in the 2D plane of $p_{T}=\left|\vec{p}_{T}(e)+\vec{p}_{T}\left(\not \mathbb{F}_{T}\right)\right|$ and transverse mass of the electron and missing transverse energy $m_{T}\left(e, \notin_{T}\right)$, which dramatically diminish jet backgrounds while preserving $80 \%$ of signal events. $Z \rightarrow e e$ backgrounds are reduced using a mass window cut. Of the 504 data events remaining, approximately 190 are due to backgrounds dominated by jet production. Backgrounds are evaluated by relaxing some of the selection and identification cuts and extrapolating background into the signal region. This technique significantly outperforms traditional methods using average rate of misidentifications for generic jets ("fake rates"). While this measurement is still dominated by statistical uncertainty, systematic error is comparable to measurements in electron and muon channels. The results is $264 \pm 23$ (stat) \pm 14 (syst) \pm 15 (lumi) pb and is in good agreement with the NNLO expectation ${ }^{5}$ of $251.3 \pm 5.0$ pb. Figure 3a shows the in- 
variant mass of the $e \tau \mathbb{F}_{T}$ system. As a cross-check, we drop the opposite sign requirement and a cut selecting only 1- and 3-prong hadronic tau candidates to show the characteristic two-horned tau shape in the tau track multiplicity plot in Fig. 3b).
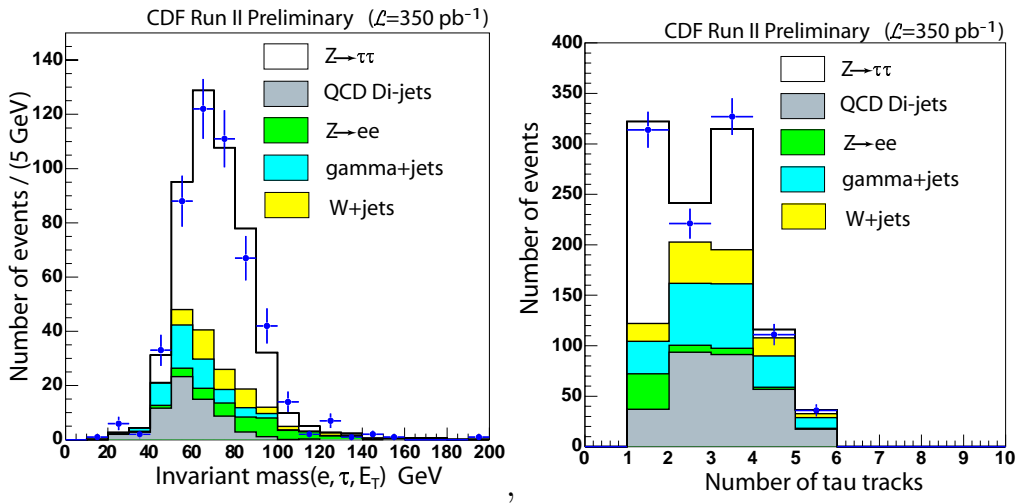

Figure 3. a): Invariant mass of the $e-\tau-\mathbb{F}_{T}$ system in $Z \rightarrow \tau \tau$ candidate events; b) number of tracks $N_{t r k}$ in tau candidates after dropping opposite-sign and $N_{t r k}=1,3$ cuts.

\section{Conclusions}

CDF has a rich program aimed at precision verification of the SM at the energy frontier. These measurements are important not only for better understanding of the SM, but also for shedding light on the yet to be discovered physics, e.g. Higgs sector or new heavier scale new physics. Improvements in the PDF uncertainties propagate to the sensitivity of the future LHC experiments.

\section{References}

1. K. Hagiwara, S. Ishihara, R. Szalapeski and D. Zeppenfeld, Phys. Rev. D48, $2182(1993)$

2. J.M. Campbell and R.K. Ellis, Phys. Rev. D60, 113006 (1999).

3. U. Baur and E.L. Berger,Phys. Rev. D41, 1476 (1990).

4. U. Baur and E.L. Berger,Phys. Rev. D47, 4889 (1993).

5. R. Harlander and W. Kilgore, Phys. Rev. Lett. 88, 201801 (2002). 